\title{
Kluyveromyces marxianus CCT7735: uma revisão sobre os mecanismos de respostas ao estresse por etanol com aplicação na produção de biocombustível
}

Amanda Fernandes Brito

Mestre em Microbiologia Agrícola pela Universidade Federal de Viçosa

E-mail: amanda_fernandis@yahoo.com.br 


\section{Resumo}

A produção do etanol como combustível é uma alternativa promissora a utilização dos combustíveis fósseis. Nesse contexto, os resíduos agroindustriais têm despontado como matérias-primas capazes de aumentar a produção de etanol sem competir com a produção de alimentos. Assim, tanto o soro de queijo, quanto subprodutos lignocelulósicos podem ser utilizados como substratos para a produção de etanol. No entanto, a levedura Saccharomyces cerevisiae é incapaz de metabolizar a lactose presente no soro de queijo e de crescer nas elevadas temperaturas requeridas no processo de Sacarificação e Fermentação Simultâneas (SSF) de biomassa lignocelulósica. Por outro lado, Kluyveromyces marxianus CCT7735 é uma linhagem desta levedura, que apresenta potencial para produzir etanol tanto a partir de soro de queijo quanto de subprodutos lignocelulósicos. Isto porque ela fermenta lactose e cresce em altas temperaturas. Todavia, ela tem o seu crescimento inibido em altas concentrações de etanol. Portanto, para viabilizar a produção de etanol, torna-se necessário compreender os mecanismos de resposta ao estresse por etanol em $K$. marxianus. Sendo assim, este trabalho teve como objetivo descrever e analisar os principais mecanismos de resposta ao estresse por etanol em leveduras, descritos na literatura, através de uma revisão bibliográfica, a fim de compreender como $K$. marxianus responde ao estresse etanólico. Nesse sentido, a abordagem utilizada nesta pesquisa foi do tipo exploratório, descritivo e bibliográfico. Desta forma, os artigos obtidos a partir das bases de dados eletrônicos foram analisados e sistematizados de acordo com a sua relevância para a compreensão dos mecanismos de respostas ao estresse por etanol em leveduras. A partir da análise dos mecanismos envolvidos na tolerância ao etanol descritos na literatura concluiu-se que eles contribuem para o entendimento das respostas moleculares ao estresse etanólico em $K$. marxianus. Entretanto, devido à complexidade destes mecanismos torna-se necessário fazer um estudo mais detalhado a nível molecular a fim de compreendê-los melhor.

Palavras-chave: Etanol. Resíduos agroindustriais. Estresse. 


\section{Abstract}

The production of ethanol as a fuel is a promising alternative to the use of fossil fuels. In this context, agro-industrial waste has emerged as raw materials capable of increasing ethanol production without competing with food production. Thus, both whey and lignocellulosic byproducts can be used as substrates for the production of ethanol. However, yeast Saccharomyces cerevisiae is unable to metabolize the lactose present in cheese serum and to grow at the high temperatures required in the Simultaneous Saccharification and Fermentation (SSF) process of lignocellulosic biomass. On the other hand, Kluyveromyces marxianus CCT7735 is a strain of this yeast, which has the potential to produce ethanol both from cheese whey and from lignocellulosic byproducts. This is because it ferments lactose and grows at high temperatures. However, it has its growth inhibited at high concentrations of ethanol. Therefore, to make viable the production of ethanol, it becomes necessary to understand the mechanisms of ethanol stress response in K. marxianus. Thus, this work aimed to describe and analyze the main mechanisms of ethanol stress response in yeasts, described in the literature, through a literature review, in order to understand how $\mathrm{K}$. marxianus responds to ethanolic stress. In this sense, the approach used in this research was exploratory, descriptive and bibliographic. In this way, the articles obtained from the electronic databases were analyzed and systematized according to their relevance to the understanding of the mechanisms of ethanol stress responses in yeasts. From the analysis of the mechanisms involved in the tolerance to ethanol described in the literature it was concluded that they contribute to the understanding of the molecular responses to ethanolic stress in K. marxianus. However, due to the complexity of these mechanisms it becomes necessary to make a more detailed study at the molecular level in order to understand them better.

Keywords: Ethanol. Agroindustrial waste. Stress. 


\section{Introdução}

A produção de etanol é uma alternativa para substituir a gasolina e consequentemente diminuir as emissões de gases poluentes na atmosfera. Atualmente, boa parte do etanol produzido no mundo é obtida partir da cana-de-açúcar e do milho, o chamado etanol de primeira geração (MORALES et al., 2015). Todavia, essas matérias-primas são alimentos, o que dificulta alavancar a produção de etanol. Assim, é necessário desenvolver novas tecnologias de produção de etanol que permitam o aumento da sua produção sem competir com a produção de alimentos. Nesse sentido, a utilização de subprodutos agroindustriais, que são abundantes e de baixo custo, é uma alternativa promissora (GUO et al., 2015).

Dentre esses subprodutos, destaca-se o soro de queijo, no entanto, a tradicional levedura utilizada na fermentação alcoólica, Saccharomyces cerevisiae, é incapaz de metabolizar a lactose, e, portanto, fermentar o soro de queijo. Por outro lado, algumas linhagens de Kluyveromyces marxianus são capazes de converter eficientemente a lactose do soro de queijo em etanol (BARNETT et al., 2000). K. marxianus CCT7735, uma linhagem desta levedura, converte a lactose do soro de queijo em etanol com rendimentos próximos ao teórico, quando cultivada em alta concentração de lactose e baixo nível de oxigênio (SILVEIRA et al., 2005). As condições ótimas de produção de etanol nesse substrato foram estabelecidas posteriormente (DINIZ et al., 2014).

O etanol também pode ser produzido a partir de biomassa celulósica, por um processo conhecido como Sacarificação e Fermentação Simultâneas (SSF), que utiliza temperaturas elevadas, em torno de $50^{\circ} \mathrm{C}$. No entanto, S. cerevisiae é incapaz de crescer nessas temperaturas. Uma alternativa interessante para contornar esse problema é o uso de leveduras termotolerantes, como, por exemplo, $K$. marxianus que apresenta potencial para produzir etanol em bagaço de cana-de-açúcar pré-tratado por meio do processo SSF (SOUZA et al., 2012).

K. marxianus CCT7735 possui potencial para produzir etanol tanto a partir de soro de queijo, quanto do bagaço de cana-de-açúcar. No entanto, esta levedura tem o seu crescimento inibido em altas concentrações de etanol devido ao estresse induzido por este metabólito. Portanto, para viabilizar a produção de etanol, torna-se necessário compreender os mecanismos de resposta ao estresse por etanol em $K$. marxiaus CCT7735. Contudo, tais mecanismos são ainda desconhecidos nesta levedura. Sendo 
assim, este trabalho teve como objetivo descrever e analisar os principais mecanismos de resposta ao estresse por etanol em leveduras, descritos na literatura, através de uma revisão bibliográfica, a fim de compreender como $K$. marxianus CCT7735 responde ao estresse etanólico.

\section{Metodologia}

Este trabalho foi realizado a partir de uma revisão sistemática descritiva, desenvolvida com produção científica indexada nas seguintes bases eletrônicas de dados: PubMed- NCBI, Science Direct, Periódicos Capes e Scielo. Os descritores utilizados na pesquisa foram "etanol", "resíduos agroindustriais" e "estresse". Foram selecionados artigos publicados entre 2005 e 2015 e que abordavam como temática a produção de etanol por leveduras. E foram excluídos os artigos repetidos e os anteriores ao período determinado. Ao final da pesquisa, foram encontrados 450 artigos, porém, apenas 74 mostraram relevância sobre o assunto.

Após a seleção dos artigos conforme os critérios de inclusão previamente definidos, foram seguidos, nessa ordem, os seguintes passos: leitura exploratória; leitura seletiva e escolha do material que se adequam aos objetivos e tema deste estudo; leitura analítica e análise dos textos, finalizando com a realização de leitura interpretativa e redação do trabalho.

\section{Resultados e Discussão}

$\mathrm{Na}$ busca inicial foram encontrados 400 artigos. E após a leitura dos títulos, notou-se que alguns artigos se repetiram nas diferentes bases de dados e outros não preenchiam os critérios deste estudo. Assim, foram selecionados 150 artigos para a leitura do resumo e excluí- dos os que não diziam respeito ao propósito deste estudo. Após a leitura dos resumos, foram selecionados 74 artigos para o fichamento, e uma vez que estes preencheram os critérios inicialmente propostos foram lidos na íntegra. Todos os artigos selecionados referiam-se a publicações brasileiras e estrangeiras. A maioria das publicações apresentava idioma inglês. 
BRITO, A. F. Kluyveromyces marxianus CCT7735: investigações sobre os mecanismos de respostas ao estresse por etanol. $R$. Cientifica UBM - Barra Mansa (RJ), ano XXII, v. 19, n. 37, 2. Sem. 2017. p. 39-60.

ISSN 1516-4071

Dos artigos selecionados observou-se que nas últimas décadas, o interesse pelos biocombustíveis vem aumentando consideravelmente, pois além de ser uma fonte de energia renovável, são menos poluentes que os combustíveis fósseis (DEMIRBAS et al., 2009; MARYANA et al., 2014). O etanol, principal biocombustível produzido no mundo, é obtido substancialmente a partir da fermentação da cana-de-açúcar e do milho, todavia, essas matérias-primas também são utilizadas pela indústria de alimentos (MARYANA et al., 2014). Sendo assim, uma alternativa para aumentar a produção de etanol sem competir com a produção de alimentos é o desenvolvimento de novas tecnologias que utilizam subprodutos agroindustriais abundantes e de baixo custo como matérias-primas (GABARDO et al., 2014). Além disso, essas fontes de energia apresentam grande quantidade de açúcares potencialmente fermentáveis á etanol capaz de atender a crescente demanda por combustíveis (SAVALIYA et al., 2015).

O soro de queijo é um efluente oriundo da indústria de laticínios que promove poluição ambiental quando descartado sem tratamento prévio na natureza (SOUZA et al., 2010; KOUSHKI et al., 2012). A fermentação da lactose, abundante açúcar do soro de queijo, constitui-se uma alternativa promissora para aliar o tratamento desse efluente à obtenção de etanol (FLORÊNCIO et al., 2013). A reutilização do soro de queijo é fortemente desejável, pois pode transformar essa fonte de carbono disponível em energia renovável sem concorrência com as culturas alimentares (ARIYANTI e HADIYANTO, 2013). Porém, S. cerevisiae, a tradicional levedura da fermentação alcoólica não assimila a lactose como fonte de carbono (RUSSEL, 1986; KARGI e OZMICHI, 2006; LING, K.C, 2008). Por outro lado, algumas linhagens de $K$. marxianus são capazes de fermentar a lactose (GUIMARÃES et al., 2010; GABARDO et al., 2014).

Biomassas lignocelulósicas, são matérias-primas abundantes e de baixo custo que também podem ser utilizadas na produção de etanol, pelo processo de Sacarificação e Fermentação Simultâneas (SSF) (OLOFSSON et al., 2008; TOMÁS-PEJÓ et al., 2009; SOUZA et al., 2012; WAHONO et al., 2014;). Esse processo é conduzido em temperaturas em torno de $50^{\circ} \mathrm{C}$, que correspondem às temperaturas ótimas das enzimas celulolíticas. Entretanto, a temperatura ótima de crescimento da levedura $S$. cerevisiae é $30^{\circ} \mathrm{C}$, ou seja, o seu crescimento é inibido nas temperaturas utilizadas no processo SSF. Nesse contexto, a utilização de leveduras que são capazes de fermentar em altas temperaturas é crucial para o processo SSF. Por ser termotolerante, Kluyveromyces 
BRITO, A. F. Kluyveromyces marxianus CCT7735: investigações sobre os mecanismos de respostas ao estresse por etanol. $R$. Cientifica UBM - Barra Mansa (RJ), ano XXII, v. 19, n. 37, 2. Sem. 2017. p. 39-60.

ISSN 1516-4071

marxianus vem sendo utilizada em vários estudos de produção de etanol de segunda geração (MORENO et al., 2013; GABARDO et al., 2014). Algumas linhagens desta levedura são consideradas excelentes produtoras de etanol (CASTRO, C. R. A; ROBERTO, I. C, 2014) pois conseguem metabolizar vários tipos de açúcares, incluindo glicose, manose, galactose, xilose e arabinose (RODRUSSAMEE et al ., 2011; SIGNORI et al., 2014).

K. marxianus é uma levedura hemiascomiceta, homotálica, relacionada filogeneticamente às leveduras Kluyveromyces lactis e $S$. cerevisae (LANE; MORRISSEY, 2011). Inicialmente, $K$. marxianus foi classificada como uma levedura Crabtree negativa (FONSECA, 2008), isto é, em ambientes aeróbios ela direciona o metabolismo preferencialmente para o ciclo do ácido tricarboxílico. Todavia, há estudos mostrando que algumas linhagens produzem etanol quando cultivadas em altas concentrações de açúcar, sendo classificadas como respiro-fermentativas, ou seja, a fermentação e a respiração ocorrem simultaneamente (LANE; MORRISSEY, 2011).

Essa levedura tem sido isolada de diferentes ambientes, apresentando alta diversidade metabólica e substancial grau de polimorfismo intraespecífico (FONSECA et al., 2008). A levedura $K$. marxianus CCT 7735, isolada do ambiente de laticínios na região da Zona da Mata Mineira, denominada inicialmente $K$. marxianus UFV-3, é capaz converter a lactose do soro de queijo em etanol com rendimentos próximos ao teórico, quando cultivada alta concentração de lactose e baixo nível de oxigênio (SILVEIRA et al., 2005). As condições ótimas de produção de etanol nesse substrato foram estabelecidas posteriormente (Diniz et al., 2014).

$K$. marxianus possui os genes $L A C 12$ e $L A C 4$ que codificam a permease de lactose e a enzima $\beta$-galactosidase, respectivamente (LANE e MORRISSEY, 2010; GABARDO et al., 2014). Primeiro, a lactose é transportada do meio extracelular pela permease, em seguida, a enzima $\beta$-galactosidase catalisa a reação de hidrólise da molécula de lactose em glicose e galactose. A glicose é direcionada para a via glicolítica, enquanto a galactose é metabolizada pela via de Leloir (SYRIOPOULOS et al., 2008), formando a glicose 1-P que é convertida em glicose 6-P, que entra na via glicolítica (SEIBOTH et al., 2007). O piruvato, último intermediário da via glicolítica, dependendo das condições de cultivo pode seguir pelo ciclo do Ácido cítrico sendo totalmente oxidado a $\mathrm{CO}_{2}$ ou entrar na via fermentativa sendo reduzido a etanol (BAI et al., 2008; PFEIFFER e MORLEY, 2014). 
BRITO, A. F. Kluyveromyces marxianus CCT7735: investigações sobre os mecanismos de respostas ao estresse por etanol. $R$. Cientifica UBM - Barra Mansa (RJ), ano XXII, v. 19, n. 37, 2. Sem. 2017. p. 39-60.

ISSN 1516-4071

K. marxianus CCT 7735 também apresenta potencial para produzir etanol em bagaço de cana-de-açúcar pré-tratado por meio do processo SSF (SOUZA et al., 2012). Ao contrário de $S$. cerevisiae, $K$. marxianus e boa parte das leveduras são incapazes de sobreviver em concentrações superiores de etanol a 6\% v/v (FLEET,1990; GIL et al., 1996; PINA et al, 2004). K. marxianus CCTT 7735 apresenta forte inibição do seu crescimento na concentração de etanol de 8\% (v/v) (SILVEIRA et al., 2005). Apesar de S.cerevisiae ser mais tolerante ao etanol, o acúmulo deste metabólito durante a fermentação interfere em sua viabilidade e capacidade fermentativa (LEWIS et al., 2010; KIM et al., 2013a). Sendo assim, o efeito e as respostas adaptativas ao etanol vêm sendo amplamente investigados a fim de compreender os mecanismos de tolerância ao etanol.

As leveduras necessitam manter um ambiente intracelular adequado ao seu crescimento e funcionamento. Porém, em condições de estresse ocorre instabilidade celular, levando a flutuações no ambiente interno. Altas concentrações intracelulares de etanol inibem o crescimento celular, provocando alterações metabólicas e estruturais que levam à redução da viabilidade das leveduras durante o processo fermentativo (WANG et al., 2007; RICCI et al., 2012; BLEOANCA et al., 2013).

Inicialmente, o etanol realiza ligação com os grupos polares dos fosfolipídios presentes nas membranas, reduzindo assim a quantidade de água presente nesta estrutura, o que induz a sua desidratação (PATRA et al., 2006). A interação deste álcool com os lipídios da membrana celular interfere na integridade desta estrutura, aumentando a sua permeabilidade e fluidez, o que promove a dissipação do potencial eletroquímico de membrana com a subsequente acidificação intracelular (MA e LIU, 2010a; VANEGAS et al., 2012; PASCHOS et al., 2015). Além disso, este metabólito induz a redução da espessura da membrana (DICKEY et al., 2010), afetando o transporte de pequenas moléculas tais como aminoácidos e açúcares (ALEXANDRE et al., 2001; LEI et al., 2007; BLEOANCA et al., 2013). O etanol estimula ainda a formação espécies reativas de oxigênio que causam danos ao DNA (HONG et al., 2010; KAHR et al., 2011; SASANO, 2012a), lipídios e proteínas (AUESUKAREE et al., 2009; ANDERSON et al., 2012). Ele também inativa enzimas importantes, como a hexoquinase e piruvato quinase, pela mudança conformacional da estrutura destas proteínas (HONG et al., 2010; DOĞAN et al., 2014; PASCHOS et al., 2015). 
BRITO, A. F. Kluyveromyces marxianus CCT7735: investigações sobre os mecanismos de respostas ao estresse por etanol. $R$. Cientifica UBM - Barra Mansa (RJ), ano XXII, v. 19, n. 37, 2. Sem. 2017. p. 39-60.

ISSN 1516-4071

Porém, quando as leveduras são submetidas ao estresse por etanol, elas conseguem desenvolver várias respostas moleculares para reparar os danos e proteger as estruturas celulares dos efeitos induzidos pelo etanol, a fim de reestabelecer a homeostase intracelular. Devido à exposição aos efeitos tóxicos do etanol, as leveduras reorganizam as atividades celulares para se adaptarem a essa condição de estresse, garantindo assim a sua sobrevivência (ARAKI et al., 2009; PLAZA et al., 2013; PASCHOS et al., 2015). As estratégias utilizadas pelas leveduras para tolerar os efeitos tóxicos do etanol incluem a regulação da expressão gênica, remodelamento de estruturas celulares e síntese de metabólitos (YU et al., 2012; LI et al., 2012). As principais respostas da levedura $S$. cerevisiae ao estresse por etanol envolvem as proteínas do choque térmico (HSPs), bem como as chaperonas SSA e APJI, que são altamente expressas nesta condição de estresse. As HSPs pertencem à classe das chaperonas, enzimas que atuam auxiliando o enovelamento proteico, assegurando assim a conformação funcional das proteínas após os processos de desnaturação provocados pelo etanol. (MCCLELLAN et al., 2007; GONG et al., 2009; NICOLAOU et al., 2010). Desta forma espera-se que $K$. marxianus ative diversas respostas celulares, incluindo enzimas metabólicas, antioxidantes, síntese de metabólitos e chaperonas moleculares a fim de minimizar os danos intracelulares e reestabelecer a homeostase.

As vias de transdução de sinal que regulam a atividade dos fatores de transcrição Hsf1, Msn2 e Msn4 são requeridas na proteção contra o estresse por etanol (BERRY et al., 2011). Hsf1 é um fator de transcrição que induz a expressão de genes que codificam proteínas do choque térmico, HSEs (ARAKI et al., 2009; NOGUCHI et al., 2011). O etanol estimula a translocação de Msn2 e Msn4 do citoplasma para o núcleo, levando a ativação da transcrição dos genes associados aos elementos de respostas ao estresse (WATANABE et al., 2009; NOGUCHI et al., 2011), dos genes que codificam as proteínas de choque térmico e dos genes cujos produtos controlam a acumulação de trealose (VOORST et al., 2006; BLEOANCA et al., 2013). Assim, os genes de síntese da trealose (TPS1, TPS2, TSL1, PGM2, e UPG1) são regulados positivamente sob o estresse por álcool, elevando assim a quantidade de trealose no meio. Esse metabólito ajuda no dobramento correto de proteínas, inibe a desnaturação de proteínas, bem como diminui a permeabilidade da membrana (DING et al., 2010; MAHMUD et al., 2012; ZHENG et al., 2013; WANG et al., 2014). As respostas ao estresse por etanol envolvendo os fatores transcricionais HSF1 e Msn2/Msn4 são fundamentais para 
BRITO, A. F. Kluyveromyces marxianus CCT7735: investigações sobre os mecanismos de respostas ao estresse por etanol. $R$. Cientifica UBM - Barra Mansa (RJ), ano XXII, v. 19, n. 37, 2. Sem. 2017. p. 39-60.

ISSN 1516-4071

estabilizar e prevenir a agregação de proteínas desnaturadas e mal dobradas (BLEOANCA et al., 2013).

Neste contexto, a resposta transcricional possibilita a identificação de genes de resposta ao etanol em $K$. marxianus. Na literatura, a alteração na expressão de genes relacionados à organização da parede celular, membrana, biogênese, biossíntese de proteínas e trealose, bem como aqueles envolvidos no metabolismo de aminoácidos, nucleotídeos, lipídios, ácidos graxos e ergosterol são descritos como responsáveis por conferir a levedura $S$. cerevisae tolerância ao etanol (TEIXEIRA et al., 2009; YOSHIKAWA et al., 2009; PÉREZ-GALLARDO et al., 2013). E desta forma, eles também devem estar relacionados à tolerância ao etanol em $K$. marxianus, podendo ser alvo de estudos posteriores. Além disso, a ativação de genes relacionados à resposta ao estresse ambiental (ESR) induz a expressão dos genes associados à geração de energia, tais como genes que codificam enzimas glicolíticas (BERRY et al., 2011). Por outro lado, os genes envolvidos no crescimento celular, síntese de RNA mensageiro e de proteínas são reprimidos (STANLEY et al., 2010). Esse fator de resposta coincide com os relatos de estudo anteriores de que altas concentrações de etanol inibem o crescimento de $K$. marxianus.

Os mecanismos de tolerância ao etanol em leveduras são complexos e envolvem múltiplos genes e, embora a expressão de vários genes tenha sido associada às linhagens de leveduras $S$. cerevisiae resistentes ao etanol, é importante quantificar a expressão desses genes em $K$. marxianus para entender a relevância dos mesmos durante a tolerância ao etanol (LIU et al., 2009; MA e LIU, 2010b). A análise da expressão gênica permite compreender as respostas transcricionais ocorridas nas células devido às variações ambientais (PUROHIT et al., 2015).

Os danos provocados pelo etanol na membrana de leveduras, leva a mudança na composição fosfolipídica desta estrutura (HENDERSON et al., 2011), aumentando o conteúdo de ácidos graxos insaturados e ergosterol para estabilizar a sua fluidez (ZHAO, 2009; VANEGAS et al., 2012). Em S. cerevisiae, os ácidos graxos monoinsaturados palmitoléico e oleico são sintetizados para compensar os danos causados pelo estresse por etanol, conferindo tolerância às leveduras (YOU et al., 2003; AGUILERA et al., 2006; LEWIS et al., 2010). O ergosterol, principal esterol da membrana de $S$. cerevisiae (HENDERSON et al., 2011), preserva a integridade estrutural da membrana em condições de estresse ambiental (DICKEY et al., 2010). 
BRITO, A. F. Kluyveromyces marxianus CCT7735: investigações sobre os mecanismos de respostas ao estresse por etanol. $R$. Cientifica UBM - Barra Mansa (RJ), ano XXII, v. 19, n. 37, 2. Sem. 2017. p. 39-60.

ISSN 1516-4071

Essa molécula interage com os lipídeos, formando uma fase liquida ordenada, modulando a interação do etanol com a membrana, protegendo-a dos efeitos deletérios dos álcoois (VANEGAS et al., 2010). Nesse sentido, espera-se que K. marxianus sob estresse por etanol promova alterações moleculares na membrana com o intuito de reorganizar a sua bicamada lipídica impedindo assim a redução induzida pelo etanol na espessura desta estrutura. Portanto, essas modificações devem ocorrer para manter a espessura adequada da membrana, conferindo assim, um fator de sobrevivência celular diante a toxicidade do etanol.

Embora os estudos presentes na literatura contribuam para a compreensão da tolerância ao etanol em $K$. marxianus, esses mecanismos são complexos, e as respostas adaptativas a este metabólito ainda não são totalmente compreendidas. Entretanto, estudos recentes sobre o perfil metabólico de leveduras sob estresse etanólico, tem permitido avaliar as mudanças intracelulares que ocorrem nessas condições. Sendo assim, a abordagem metabolômica permite identificar metabólitos associados à resposta ao estresse por etanol em $K$. marxianus CCT7735. E assim, tais informações podem contribuir para elucidar o processo de tolerância ao etanol nesta levedura.

Segundo LI et al., (2012), o estresse por etanol causa impactos no perfil dos metabólitos relacionados ao metabolismo de carboidratos, lipídeos e aminoácidos. LOURENÇO et al, (2013) também identificou alterações no metaboloma de $S$. cerevisiae sob estresse por etanol. Neste artigo o acúmulo de aminoácidos e intermediários do ciclo de Krebs foi relacionado com a proteção celular durante o estresse. Além disso, a análise de leveduras mutantes, S. cerevisiae BY4742, sob estresse por etanol demonstrou que os metabólitos, prolina, valina e inositol contribuem para a tolerância destas leveduras ao etanol (OHTA et al., 2015). Neste sentido, devem ocorrer mudanças nos níveis de ácidos graxos e aminoácidos, associadas à tolerância ao etanol $K$. marxianus.

Em S. cerevisiae, a prolina exerce papel importante na sua adaptação ao etanol (SEKINE et al., 2007; YOSHIKAWA et al., 2009; LOURENÇO et al., 2013). Este aminoácido promove a estabilidade das membranas, inibe a agregação de proteínas e elimina as espécies reativas de oxigênio (ROS) (DING et al., 2009; SASANO, 2012ab). Portanto, pode-se considerar que o aminoácido prolina tem seus níveis aumentados durante o estresse por etanol em $K$. marxiaus, contribuindo portanto, para aumentar a estabilidade das membranas, prevenir a agregação proteica durante o redobramento 
BRITO, A. F. Kluyveromyces marxianus CCT7735: investigações sobre os mecanismos de respostas ao estresse por etanol. $R$. Cientifica UBM - Barra Mansa (RJ), ano XXII, v. 19, n. 37, 2. Sem. 2017. p. 39-60.

proteico, bem como eliminar as espécies reativas de oxigênio impedindo seu acúmulo intracelular.

\section{Conclusão}

A análise dos mecanismos envolvidos na tolerância ao etanol descritos na literatura tem contribuído para o entendimento das respostas moleculares ao estresse etanólico em $K$. marxianus CCT 7735. Entretanto, esses mecanismos são complexos e dependem de várias interações celulares. Portanto, torna-se necessário fazer um estudo mais detalhado a nível molecular a fim de compreendê-los melhor.

\section{Referências}

AGUILERA, F.; PEINADO, R.A.; MILLÁN, C.; ORTEGA, J.M.; MAURICIO, J. C. Relation ship between ethanol tolerance, H+-ATPase activity and the lipid composition of the plasma membrane in different wine yeast strains. International Journal of Food Microbiology, v. 110, p. 34-42, 2006.

ALEXANDRE, H.; ANSANAY-GALEOTE, V.; BLONDIN, S.D.B. Global gene expression during short-term ethanol stress in Saccharomyces cerevisiae. FEBS Letters, v. 498, p. 98-103, 2001.

ANDERSON, M. J.; BARKER, S. L.; BOONE, C.; MEASDAY, V. Identification of RCN1 and RSA3 as ethanol-tolerant genes in Saccharomyces cerevisiae using a high copy barcoded library. FEMS Yeast Research, v. 12, p.48-60, 2012.

ARAKI, Y.; WU, H.; KITAGAKI, H.; AKAO, T.; TAKAGI, T e SHIMOI, H. Ethanol stress stimulates the $\mathrm{Ca}^{2+-}$ mediated calcineurin/Crz1 pathway in Saccharomyces cerevisiae. Journal of Bioscience and Bioengineering, v. 107, p. 1-6, 2009.

ARIYANTI, D e HADIYANTO, H. Ethanol Production from Whey by Kluyveromyces marxianus in Batch Fermentation System: Kinetics Parameters Estimation. Bulletin of Chemical Reaction Engineering E Catalysis, v. 7, p. 179-184, 2013. 
BRITO, A. F. Kluyveromyces marxianus CCT7735: investigações sobre os mecanismos de respostas ao estresse por etanol. $R$. Cientifica UBM - Barra Mansa (RJ), ano XXII, v. 19, n. 37, 2. Sem. 2017. p. 39-60.

AUESUKAREE, C.; DAMNERNSAWAD, A.; KRUATRACHUE, M.; POKETHITIYOOK, P.; BOONCHIRD, C.; KANEKO, Y.; HARASHIMA, S. Genome-wide identification of genes involved in tolerance to various environmental stresses in Saccharomyces cerevisiae. Journal Applied Genetic, v. 50, p. 301-310, 2009.

BAI, F. W.; ANDERSON, W. A.; MOO-YOUNG, M. Ethanol fermentation technologies from sugar and starch feedstocks. Biotechnology Advances, v. 26, p. 89-105, 2008.

BERRY, D. B.; GUAN, Q.; HOSE, J.; HAROON, S.; GEBBIA, M.; HEISLER2, L.E.; NISLOW, C.; GIAEVER, G.; GASCH, A.P. Multiple means to the same end: the genetic basis of acquired stress resistance in yeast. PLOS Genetics, v. 7, p. 1-11, 2011.

BLEOANCA, I.; SILVA, A.R.C.; PIMENTEL, C.; POUSADA, C. R.;

MENEZES, R. A. Relationship between ethanol and oxidative stress in laboratory and brewing yeast strains. Journal of Bioscience and Bioengineering, v. 116, p. 697-705, 2013.

CASTRO, R. C. A.; ROBERTO, I. C. Selection of a thermotolerant Kluyveromyces marxianus strain with potential application for cellulosic ethanol production by simultaneous saccharification and fermentation. Applied Biochemistry and Biotechnology, v. 172, p. 1553-1564, 2014.

DICKEY, A. N.; YIM, W. S.; FALLER, R. Using ergosterol to mitigate the deleterious effects of ethanol on bilayer structure. The Journal of Physical Chemistry B, v.113, p. 2388-2397, 2010.

DING, J.; HUANG, X.; ZHANG, L.; ZHAO, N.; YANG, D.; ZHANG, K. Tolerance and stress response to ethanol in the yeast Saccharomyces cerevisiae. Applied Microbiology and Biotechnology, v. 85, p.253-263, 2009.

DING, M. Z.; LI, B. Z.; CHENG, J.S.; YUAN, Y. J. Metabolome analysis of differential responses of diploid and haploid yeast to ethanol stress. OMICS, v.14, p. 553-561, 2010.

DINIZ, R. H. S.; RODRIGUES, M. Q. R. B.; FIETTO, L.G.; PASSOS, F.M.L.; SILVEIRA, W. B. Optimizing and validating the production of ethanol from cheese 
BRITO, A. F. Kluyveromyces marxianus CCT7735: investigações sobre os mecanismos de respostas ao estresse por etanol. $R$. Cientifica UBM - Barra Mansa (RJ), ano XXII, v. 19, n. 37, 2. Sem. 2017. p. 39-60.

whey permeate by Kluyveromyces marxianus UFV-3. Biocatalysis and Agricultural Biotechnology, v.3, p.111-117, 2014.

DOĞAN, A.; DEMIRCI, S.; AYTEKIN, A. O.; ŞAHIN, F. Improvements of tolerance to stress conditions by genetic engineering in Saccharomyces cerevisiae during ethanol production. Applied Biochemistry and Biotechnology, v.174, p.28-42, 2014.

FLORÊNCIO, I. M.; FLORENTINO, E. R.; SILVA, F. L. H.; MARTINS, R. S.; CAVALCANTI, M. T.; GOMES, J. Production of ethanol from industrial whey. Revista Basileira Engenharia Agricola e Ambiental, v.17, p. 1088-1092, 2013.

FONSECA, G. G.; HEINZLE, E.; WITTMANN, C.; GOMBERT, A. K. The yeast Kluyveromyces marxianus and its biotechnological potential. Applied Microbiology and Biotechnology, v. 79, p. 339-354, 2008.

GABARDO, S.; RECH, R.; ROSA, C. A.; AYUB, M. A. Z. Dynamics of ethanol production from whey and whey permeate by immobilized strains of Kluyveromyces marxianus in batch and continuous bioreactors. Renewable Energy, v. 69, p. 89-96, 2014.

GIL, J. V.; JIMENEZ, M. M.; PASTOR, A.; HUERTA, T. Aroma compounds in wine as influenced by apiculate yeasts. Journal of Food Science, v. 61, p. 1247-1250, 1996.

GONG, Y.; KAKIHARA, Y.; KROGAN, N.; GREENBLAT, T. J.; EMILI, A.; ZHANG, Z.; HOURY, W. A. An atlas of chaperone-protein interactions in Saccharomyces cerevisiae: implications to protein folding pathways in the cell. Molecular Systems Biology, v. 5, p. 1-14, 2009.

GUIMARÃES, P. M. R.; TEIXEIRA, J. A.; DOMINGUES. L. Fermentation of lactose to bio-ethanol by yeasts as part of integrated solutions for the valorization of cheese whey. Biotechnology Advances, v. 28, p. 375-384, 2010.

HENDERSON, C.M.; LOZADA-CONTRERAS, M.; NARAVANE, Y.; LONGO, M. L.; BLOCK, D. E. Analysis of major phospholipid species and ergosterol in fermenting industrial yeast strains using atmospheric pressure ionization ion-trap mass spectrometry. Journal of Agricultural and Food Chemistry, v. 59, p. 1276112770, 2011. 
BRITO, A. F. Kluyveromyces marxianus CCT7735: investigações sobre os mecanismos de respostas ao estresse por etanol. $R$. Cientifica UBM - Barra Mansa (RJ), ano XXII, v. 19, n. 37, 2. Sem. 2017. p. 39-60.

HONG, M. F.; LEE, K-S.; YUB, B. J.; SUNGA, Y-J.; PARK, S. M.; KOO, H. M.; KWEONA, H-D.; PARK, J. C.; JINC, Y-S. Identification of gene targets eliciting improved alcohol tolerance in Saccharomyces cerevisiae through inverse metabolic engineering. Journal of Biotechnology, v. 149, p. 52-59, 2010.

KAHR, H.; HELMBERGER, S.; JÄGER, A. G. Yeast adaptation on the substrate straw. Bioenergy Technology, v. 8, p. 492-499, 2011.

KIM, S.; KIM, Y.S.; KIM, H.; JIN, I.; YOON, H. S. Saccharomyces cerevisiae KNU5377 stress response during high-temperature ethanol fermentation. Molecules and Cells, v. 35, p. 210-218, 2013a.

KIM, S.; PARK, J. M.; KIM, C.H. Ethanol production using whole plant biomass of jerusalem artichoke by Kluyveromyces marxianus CBS1555. Applied Biochemistry and Biotechnology, v. 169, p.1531-1545, 2013b.

KITAGAKI, H e TAKAGI, H. Mitochondrial metabolism and stress response of yeast: Applications in fermentation technologies. Journal of Bioscience and Bioengineering, v. 117, p. 383-393, 2014.

KOUSHKI, M.; JAFARI, M.; AZIZI, M. Comparison of ethanol production from cheese whey permeate by two yeast strains. Journal of Food Science Technology, v. 49, p. 614-619, 2012.

LANE, M. M e MORRISSEY, J. P. Kluyveromyces marxianus: A yeast emerging from its sister's shadow. Fungal Biology Reviews, v. 24, p. 17-26, 2010.

LANE, M. M.; BURKE, N.; KARREMAN, R.; WOLFE, K. H.; O'BYRNE, C. P.; MORRISSEY, J. P. Physiological and metabolic diversity in the yeast Kluyveromyces marxianus. Antonie van Leeuwenboek, v.100, p. 507-519, 2011.

LEI, J.; ZHAO, X.; GE, X.; BAI, F. Ethanol tolerance and the variation of plasma membrane composition of yeast floc populations with different size distribution. Journal of Biotechnology, v. 131, p. 270-275, 2007. 
BRITO, A. F. Kluyveromyces marxianus CCT7735: investigações sobre os mecanismos de respostas ao estresse por etanol. $R$. Cientifica UBM - Barra Mansa (RJ), ano XXII, v. 19, n. 37, 2. Sem. 2017. p. 39-60.

LEWIS, J.A.; ELKON, I.M.; McGEE, M.A.; HIGBEE, A.J.; GASCH, A.P. Exploiting natural variation in Saccharomyces cerevisiae to identify genes for increased ethanol resistance. Genetics, v. 186, p.1197-205, 2010.

LI, H.; MA, M.L.; LUO, S.; ZHANG, R.M.; HAN, P.; HU, W. Metabolic responses to ethanol in Saccharomyces cerevisiae using a gas chromatography tandem mass spectrometry-based metabolomics approach. The International Journal of Biochemistry ${ }^{\circ}$ Cell Biology, v. 44, p. 1087- 1096, 2012.

LIU, Z. L.; MA, M.; SONG, M. Evolutionarily engineered ethanologenic yeast detoxifies lignocellulosic biomass conversion inhibitors by reprogrammed pathways. Molecular Genetics and Genomics, v. 282, p. 233-244, 2009.

LOURENÇO, A. B.; ROQUE, F.C.; TEIXEIRA, M. C.; ASCENSO, J. R.; CORREIA, I. S. Quantitative 1 H-NMR-Metabolomics Reveals Extensive Metabolic Reprogramming and the Effect of the Aquaglyceroporin FPS1 in Ethanol-Stressed Yeast Cells. PLOS ONE, v. 8, p. 1-12, 2013.

MA, M e LIU, L.Z. Quantitative transcription dynamic analysis reveals candidate genes and key regulators for etanol tolerance in Saccharomyces cerevisiae. BMC Microbiology, v. 10, p. 1471-2180, 2010b.

MA, M e LIU, Z. L. Mechanisms of ethanol tolerance in Saccharomyces cerevisiae. Applied Microbiology Biotechnology, v. 87 p.829-845, 2010a.

MAHMUD, S. A.; HIRASAWA, T.; FURUSAWA, C.; YOSHIKAWA, K.; SHIMIZU, H. Understanding the mechanism of heat stress tolerance caused by high trehalose accumulation in Saccharomyces cerevisiae using DNA microarray. Journal of Bioscience and Bioengineering, v. 113, p. 526-528, 2012.

MARYANA, R.; MA'RIFATUNB, D.; WHENI, I. A.; SATRIYO, K. W; RIZALA, W. A. Alkaline Pretreatment on Sugarcane Bagasse for Bioethanol Production. Energy Procedia, v. 47, p. 250-254, 2014. 
BRITO, A. F. Kluyveromyces marxianus CCT7735: investigações sobre os mecanismos de respostas ao estresse por etanol. $R$. Cientifica UBM - Barra Mansa (RJ), ano XXII, v. 19, n. 37, 2. Sem. 2017. p. 39-60.

MCCLELLAN, A. J.; XIA, Y.; DEUTSCHBAUER, A. M.; DAVIS, R.W.; GERSTEIN, M.; FRYDMAN, J. Diverse cellular functions of the Hsp90 molecular chaperone uncovered using systems approaches. Cell, v. 5, p. 121-135, 2007.

NICOLAOU, S. A.; GAIDA, S. M.; PAPOUTSAKIS, E.T. A comparative view of metabolite and substrate stress and tolerance in microbial bioprocessing: From biofuels and chemicals, to biocatalysis and bioremediation. Metabolic Engineering, v. 12, p.307331, 2010.

NOGUCHI, C.; WATANABE, D.; ZHOU, Y.; AKAO, K.; SHIMOI, H.

Association of Constitutive Hyperphosphorylation of Hsflp with a Defective Ethanol Stress Response in Saccharomyces cerevisiae Sake Yeast Strains. Applied and Environmental Microbiology, p. 385-392, 2011.

OHTA, E.; NAKAYAMA, Y.; MUKAI, Y.; BAMBA, T e FUKUSAK, E. Metabolomic approach for improving ethanol stress tolerance in Saccharomyces cerevisiae. Journal of Bioscience and Bioengineering, v. 15, p. 1-7, 2015.

PASCHOS, T.; XIROS, C.; CHRISTAKOPOULOS, P. Ethanol effect on metabolic activity of the ethalogenic fungus Fusarium oxysporum. BMC Biotechnology, v.15, p.112, 2015.

PATRA, M.; SALONEN, E.; TERAMA, E.; VATTULAINEN, I.; FALLER, R.; LEE, B. W.; HOLOPAINEN, J e KARTTUNEN, M. Under the Influence of Alcohol: The Effect of Ethanol and Methanol on Lipid Bilayers. Biophysical Journal, v. 90, p. 1121-1135, 2006.

PÉREZ-GALLARDO, R. V.; BRIONES, L.S.; DÍAZ-PÉREZ, A. L.; GUTIÉRREZ, S.; RODRIGUEZ-ZAVALA, J.S.; CAMPOS-GARCIA, J. Reactive oxygen species production induced by ethanol in Saccharomyces cerevisiae increases because of a dysfunctional mitochondrial iron-sulfur cluster assembly system. FEMS, v. 13, p. 804-819, 2013.

PFEIFFER, T e MORLEY, A. An evolutionary perspective on the Crabtree effect. Frontiers in Molecular Biosciences, v. 1, p. 1-6, 2014. 
BRITO, A. F. Kluyveromyces marxianus CCT7735: investigações sobre os mecanismos de respostas ao estresse por etanol. $R$. Cientifica UBM - Barra Mansa (RJ), ano XXII, v. 19, n. 37, 2. Sem. 2017. p. 39-60.

ISSN 1516-4071

PHAM, T. K e WRIGHT, P. C. Proteomic Analysis of Calcium AlginateImmobilized Saccharomyces cerevisiae under High-Gravity Fermentation Conditions. Journal Proteome Research, v.2, p. 515-25, 2008.

PINA, C.; ANTÓNIO, J.; HOGG, T. Ethanol tolerance of five non-Saccharomyces wine yeasts in comparison with a strain of Saccharomyces cerevisiae - influence of different culture conditions. Food Microbiology, v. 21, p. 439-447, 2004.

PLAZA, M, A.; MATHIS, A. G.; CISNEROS, R. N.; GUERRERO, M. C.; MANZO-ÁVALOS, S.; HERNANDEZ, J. C. G.; SAAVEDRA-MOLINA, A. Respiratory capacity of the Kluyveromyces marxianus yeast isolated from the mescal process during oxidative stress. World Journal Microbiology and Biotechnology, v. 29, p.1279-1287, 2013.

PURIA, R.; MANNAN, M. A.; CHOPRA-DEWASTHALY, R.; GANESAN, K. Critical role of RPI1 in the stress tolerance of yeast during ethanolic fermentation. FEMS Yeast Research, v. 9, p. 1161-1171, 2009.

PUROHIT, G.K.; MAHANTY, A.; MOHANTY, B.P.; MOHANTY, S. Evaluation of housekeeping genes as references for quantitative real-time PCR analysis of gene expression in the murrel Channa striatus under high-temperature stress. Fish Physiology and Biochemistry, p. 1-11, 2015.

RICCI. M.; AGGRAVI, M.; BONECHI, C.; MARTINI, S.; ALOISI, A.M.; ROSSI, C. Metabolic response to exogenous ethanol in yeast: An in vivo statistical total correlation NMR spectroscopy approach. Journal Bioscience, v. 37, p. 749-755, 2012.

RODRUSSAMEE, N.; LERTWATTANASAKUL, N.; HIRATA, K.; SUPRAYOGI.; LIMTONG, S.; KOSAKA, T.; YAMADA, M. Growth and ethanol fermentation ability on hexose and pentose sugars and glucose effect under various conditions in thermotolerant yeast Kluyveromyces marxianus. Applied Microbiology and Biotechnology, v. 90, p.1573-1586, 2011.

SASANO, Y.; HAITANI, Y.; HASHIDA, K.; OHTSU, I.; SHIMA, J.; TAKAGI, H. Enhancement of the proline and nitric oxide synthetic pathway improves fermentation ability under multiple baking-associated stress conditions in industrial baker's yeast. Microbial Cell Factories, v.11, p.1-8, 2012a. 
BRITO, A. F. Kluyveromyces marxianus CCT7735: investigações sobre os mecanismos de respostas ao estresse por etanol. $R$. Cientifica UBM - Barra Mansa (RJ), ano XXII, v. 19, n. 37, 2. Sem. 2017. p. 39-60.

SASANO, Y.; HAITANI, Y.; OHTSU, I.; SHIMA, J.; TAKAGI, H. Proline accumulation in baker's yeast enhances high-sucrose stress tolerance and fermentation ability in sweet dough. International Journal of Food Microbiology, v. 152, p. 40-43, 2012b.

SEIBOTH, B.; PAKDAMAN, B. S.; HARTL, L.; KUBICEK, C.P. Lactose metabolism in filamentous fungi: how to deal with an unknown substrate. Fungal Biology Reviews, v. 21, p.42 - 48, 2007.

SENGUPTA, S.; LAHIRI, S.; BANERJEE, S.; BASHISTHA, B.; GHOSH, A.K. Arginine mediated purification of trehalose-6-phosphate synthase (TPS) from Candida utilis: Its characterization and regulation. Biochimica et Biophysica Acta, v. 1810, p.13461354, 2011.

SEKINE, T.; KAWAGUCHI, A.; HAMANO, Y e TAKAGI, H. Desensitization of Feedback Inhibition of the Saccharomyces cerevisiae -Glutamyl Kinase Enhances Proline Accumulation and Freezing Tolerance. Applied and Environmental Microbiology, v.73, p. 4011-4019, 2007.

SIGNORI, L.; PASSOLUNGHI, S.; RUOHONEN, L.; PORRO, D.; BRANDUARDI, P. Effect of oxygenation and temperature on glucose-xylose fermentation in Kluyveromyces marxianus CBS712 strain. Microbial Cell Factories, v, 51, p. 1-13, 2014.

SILVEIRA, W. B.; PASSOS, F. J. V.; MANTOVANI, H. C.; PASSOS, F. M. L. Ethanol production from cheese whey permeate by Kluyveromyces marxianus UFV-3: A flux analysis of oxido-reductive metabolism as a function of lactose concentration and oxygen levels. Enzyme and Microbial Technology, v. 36, p. 930-936, 2005.

SOUZA, C. J. A.; COSTA, D. A.; RODRIGUES, M. Q. R. B.; SANTOS, A. F.; LOPES, M. R.; ABRANTES, A. B. P.; COSTA, P. S.; SILVEIRA, W. B.; PASSOS, F. M. L.; FIETTO, L. G. The influence of presaccharification, fermentation temperature and yeast strain on etanol production from sugarcane bagasse. Bioresourse and Biotecnology, v. 109, p. 63-69, 2012. 
BRITO, A. F. Kluyveromyces marxianus CCT7735: investigações sobre os mecanismos de respostas ao estresse por etanol. $R$. Cientifica UBM - Barra Mansa (RJ), ano XXII, v. 19, n. 37, 2. Sem. 2017. p. 39-60.

STANLEY, D.; BANDARA, A.; FRASER, S.; CHAMBERS. P. J.; STANLEY, G.A. The ethanol stress response and ethanol tolerance of Saccharomyces cerevisiae. Journal of Applied Microbiology, v.109, p. 13-24, 2010.

SYRIOPOULOS, C.; PANAYOTAROU, A.; LAI, K.; KLAPA, M. I.

Transcriptomic analysis of Saccharomyces cerevisiae physiology in the context of galactose assimilation perturbations. Molecular BioSystems, v. 4, p. 937-949, 2008.

TAKAGI, H. Proline as a stress protectant in yeast: physiological functions, metabolic regulations, and biotechnological applications. Applied Microbiology and Biotechnology, v. 81, p. 211-223, 2008.

TAKAGI, H.; TAKAOKA, M.; KAWAGUCHI, M e KUBO, Y. Effect of L-Proline on Sake Brewing and Ethanol Stress in Saccharomyces cerevisiae. Applied and Environmental Microbiology, v.71, p. 8656-8662, 2005.

TANG, M.; WARING, A.J.; HONG, M. Trehalose-protected lipid membranes for determining membrane protein structure and insertion. Journal of Magnetic Resonance, v. 184, p. 222-227, 2007.

TEIXEIRA, M. C.; R, L. R.; MIRA, N. P; LOURENÇO, A. B.; SÁ-CORREIA, I. Genome-wide identification of Saccharomyces cerevisiae genes required for maximal tolerance to ethanol. Applied and Environment Microbiology, v. 75, p. 5761-5772, 2009.

VANEGAS, J. M.; CONTRERAS, M. F.; FALLER, R.; LONGO, M. L. Role of unsaturated lipid and ergosterol in ethanol tolerance of model yeast biomembranes. Biophysical Journal, v.102, p. 507-16, 2012.

VANEGAS, J. M.; FALLER, R.; LONGO, M.L. Influence of ethanol on lipid/sterol membranes: phase diagram construction from AFM imaging. Langmuir Letter, v. 26, p. 10415-10418, 2010.

VOORST, F. V.; HOUGHTON-LARSEN, J.; JONSON, L.; KIELLANDBRANDT, M.C e BRANDT, A. Genome-wide identification of genes required for growth of Saccharomyces cerevisiae under etanol stress. Yeast, v. 23, p. 351-359, 2006. 
BRITO, A. F. Kluyveromyces marxianus CCT7735: investigações sobre os mecanismos de respostas ao estresse por etanol. $R$. Cientifica UBM - Barra Mansa (RJ), ano XXII, v. 19, n. 37, 2. Sem. 2017. p. 39-60.

WANG, M.; ZHAO, J.; YANG, Z.; DUD, Z.; YANG, Z. Electrochemical insights into the etanol tolerance of Saccharomyces cerevisiae. Bioelectrochemistry, v. 71, p. 107$112,2007$.

WANG, P.M.; ZHENG, D.Q.; CHI, X.Q.; LI, O.; QIAN, C.D.; LIU, T.Z.; ZHANG, X.Y.; DU, F.G.; SUN, P.Y.; QU, A.M.; WU, X.C. Relationship of trehalose accumulation with ethanol fermentation in industrial Saccharomyces cerevisiae yeast strains. Bioresource Technology, v. 152, p.371-376, 2014.

WATANABE, M.; WATANABE, D.; AKAO, T.; SHIMOI, H. Overexpression of MSN2 in a sake yeast strain promotes ethanol tolerance and increases ethanol production in sake brewing. Journal of Bioscience Bioengineering, v. 107, p. 516-8, 2009.

YOSHIKAWA, K.; TANAKA, T.; FURUSAWA, C.; NAGAHISA, K.; HIRASAWA, T.; SHIMIZU, H. Comprehensive phenotypic analysis for identiథcation of genes affecting growth under ethanol stress in Saccharomyces cerevisiae. FEMS Yeast Research, v. 9, p. 32-44, 2009.

YOU, K. M.; ROSENFIELD, C. L.; KNIPPLE, D. C. Ethanol tolerance in the yeast saccharomyces cerevisiae is dependent on cellular oleic acid content. Applied and Environmental Microbiology, v. 69, p. 1499-1503, 2003.

YU, K.O.; JUNG, J.; RAMZI, A. B.; CHOE, S.H.; KIM, S. W.; PARK, C.; HAN, S. O. Increased ethanol production from glycerol by Saccharomyces cerevisiae strains with enhanced stress tolerance from the overexpression of SAGA complex components. Enzyme and Microbial Technology, v. 51, p. 237-243, 2012.

ZHANG, K.; TONG, M.; GAO, K.; DI, Y.; WANG, P.; ZHANG, C.; WU, X.; ZHENG, D. Genomic reconstruction to improve bioethanol and ergosterol production of industrial yeast Saccharomyces cerevisiae. Journal Industrial Microbiology and Biotechnology, v. 42, p. 207-218, 2015.

ZHAO, X. Q.; BAI, F.W. Mechanisms of yeast stress tolerance and its manipulation for efficient fuel ethanol production. Journal of Biotechnology, v. 144, p. 23-30, 2009.

ZHENG, D. Q.; LIU, T. Z.; CHEN, J.; ZHANG, K.; LI, O.; ZHU, L.; ZHAO, Y.H; WU, X. C.; WANG, P. M. Comparative functional genomics to reveal the 
BRITO, A. F. Kluyveromyces marxianus CCT7735: investigações sobre os mecanismos de respostas ao estresse por etanol. $R$. Cientifica UBM - Barra Mansa (RJ), ano XXII, v. 19, n. 37, 2. Sem. 2017. p. 39-60.

ISSN 1516-4071

molecular basis of phenotypic diversities and guide the genetic breeding of industrial yeast strains. Applied Microbiology and Biotechnology, v. 97, p. 2067-2076, 2013. 